\title{
Multi-Fractional Analysis of Molecular Diffusion in Polymer Multilayers by FRAP: A New Simulation-Based Approach
}

\author{
David Sustr, ${ }^{\dagger \neq}$ Antonín Hlaváček, ${ }^{\S}$ Claus Duschl, ${ }^{\ddagger}$ and Dmitry Volodkin*, ${ }^{\ddagger}, \|_{\odot}$ \\ ${ }^{\dagger}$ Faculty of Science, University of Potsdam, Institute of Biochemistry and Biology, Karl-Liebknecht-Str. 24-25, 14476 Potsdam-Golm, \\ Germany \\ ${ }^{\star}$ Department of Molecular and Cellular Bioanalytics, Fraunhofer Institute for Cell Therapy and Immunology (Fraunhofer IZI), Am \\ Mühlenberg 13, 14476 Potsdam-Golm, Germany \\ ${ }^{\S}$ Institute of Analytical Chemistry of the Czech Academy of Sciences, v. v. i., Veveř́ 97, Brno 602 00, Czech Republic \\ "School of Science and Technology, Nottingham Trent University, Clifton Lane, Nottingham NG11 8NS, United Kingdom
}

Supporting Information

ABSTRACT: Comprehensive analysis of the multifractional molecular diffusion provides a deeper understanding of the diffusion phenomenon in the fields of material science, molecular and cell biology, advanced biomaterials, etc. Fluorescence recovery after photobleaching (FRAP) is commonly employed to probe the molecular diffusion. Despite FRAP being a very popular method, it is not easy to assess multifractional molecular diffusion due to limited possibilities of approaches for analysis. Here we present a novel simulationoptimization-based approach (S-approach) that significantly broadens possibilities of the analysis. In the S-approach, possible

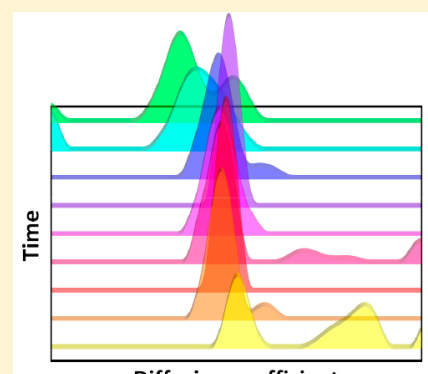

Diffusion coefficient

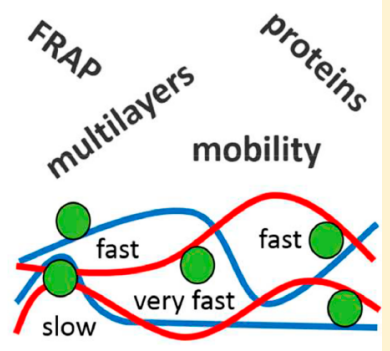

fluorescence recovery scenarios are primarily simulated and afterward compared with a real measurement while optimizing parameters of a model until a sufficient match is achieved. This makes it possible to reveal multifractional molecular diffusion. Fluorescent latex particles of different size and fluorescein isothiocyanate in an aqueous medium were utilized as test systems. Finally, the S-approach has been used to evaluate diffusion of cytochrome c loaded into multilayers made of hyaluronan and polylysine. Software for evaluation of multifractional molecular diffusion by S-approach has been developed aiming to offer maximal versatility and user-friendly way for analysis.

\section{INTRODUCTION}

Knowledge about molecular diffusion may be very valuable and provide insight into molecular interactions in living organisms, soft matter systems, and a variety of advanced functional materials. Up to now, the following methods are the most often used for the assessment of molecular diffusion: fluorescence recovery after photobleaching (FRAP), fluorescence correlation spectroscopy (FCS), and particle tracking. The FRAP approach utilizes a patterned fluorophore bleaching within an area of a fluorescent sample. The pattern diminishes as the fluorescence intensity recovers in the bleached region because of molecular diffusion. One can quantify molecular diffusion by analyzing the rate of recovery. ${ }^{1}$ In case of FCS, temporal fluctuations of fluorescence intensity within the focal volume of an objective are monitored and analyzed through autocorrelation and suitable models of diffusion. ${ }^{2}$ The last mentioned particle tracking method is based on recording the random walk of a single particle with high precision and afterward applying the mean square displacement analysis to deduce the underlying diffusion coefficient. ${ }^{3,4}$ These three main methods (FRAP, FCS, particle tracking) are complementary in many aspects and have certain limitations as well. While FRAP typically requires an observation of a certain spatial area with high fluorescence intensity and bleachable fluorophore, the FCS and the particle tracking, on the contrary, require highly diluted, stable fluorophores. In case of FRAP, the evaluation of experimental data requires some level of image processing according to the design of the experiment. Therefore, the evaluation procedures require adaptation to the specifics of the experiment, but they typically employ some general concept of FRAP data evaluation in the core. Evaluation of FCS data by the autocorrelation function may seem more straightforward, but its results are dependent on knowledge about involved phenomena and its precise parameters (e.g., a geometry of the detection volume, bleaching and photophysical dark states of a fluorophore, viscosity of local environment).,

In this work, we used the FRAP technique because it leads to a rather intuitive data evaluation and because a standard confocal microscope is sufficient for this type of experiment. The most original approach to the analysis of FRAP data was described by Axelrod et al. ${ }^{1}$ In this approach, a circular area within a sample is

Received: November 8, 2017

Revised: December 18, 2017

Published: December 19, 2017 
bleached and the recovery of fluorescence is evaluated from the temporal development of the average fluorescence intensity within the bleached area. Three characteristic points of this recovery curve or the whole recovery curve are afterward fitted by an analytical solution. The spatial distribution of the fluorescence intensity within the sample during the recovery is averaged out. It results in a loss of information but also reduction of data amount and simplification of data evaluation. This approach was mainly designed for nonscanning fluorescence microscopes and the computational power available at that time. Further development of FRAP procedures and evaluation methods have been promoted by the availability of a confocal laser scanning microscopes. There are many works extending the original evaluation approach by incorporating various phenomena into evaluation procedure: system flow, ${ }^{1,7}$ binding kinetics, ${ }^{7,8}$ various profiles of the bleached spot, ${ }^{9,10}$ arbitrary geometry of the bleached area, ${ }^{11}$ discrimination between two diffusive fractions, ${ }^{12,13}$ the profile of the laser beam, ${ }^{7}$ two-photon excitation, ${ }^{14}$ exploiting a kind of simulation for validation of the analytical approach solution, ${ }^{13,15}$ and others.

Later, several evaluation approaches taking a spatial distribution of fluorescence intensity into account were described. Typically 1D FRAP recovery profiles are fitted by a function obtained as an analytical solution of a certain case of recovery. ${ }^{16-18}$ The fitting of Gaussian is employed in this work (an A-approach). ${ }^{19}$ The utilization of the analytical solution based FRAP analysis of two-fractional ${ }^{20,21}$ or multifractional ${ }^{22,23}$ diffusion was also demonstrated. Nevertheless, an extension of the analytical approach from single-fractional to multifractional analysis makes the evaluation procedure cumbersome.

At the same time, an alternative approach was slowly emerging which employs the simulation-optimization tandem method for data evaluation. ${ }^{24-30}$ Simulations are an elegant way of identifying solution, in particular since, nowadays, the computational power of personal computers is sufficient to find solutions for such problem in a reasonably short time. The approach is widely universal-diffusion of multiple species in FRAP experiment can be easily simulated without any need to find an analytical solution of the phenomenon. Thus, it is not necessary to reduce the complexity of the system to the stage at which an analytical solution can be established (e.g., bleaching of welldefined pattern, single diffusive fraction, etc.). Despite these merits, the simulation-based approach requires quantitatively demanding calculations and handling of an ill-posed inverse problem.

In this work, we propose a new, universal, and easy-to-use tool, which allows the assessment of multifractional diffusion by employing the simulation-optimization-based approach (Sapproach). To deduce as much useful information from an experiment as possible, a shape of recovery profiles is fed into the evaluation process. Unlike in the case of the A-approach, the shape of bleached patterns is arbitrary in the proposed Sapproach. To the best of our knowledge, there is available no tool comprising all aforementioned features. We focus on providing a robust solution and a high flexibility (arbitrary bleach profile, multifractional solution, low amount of input parameters) with the S-approach. Here we compare the A- and S-approach by the assessment of single- and multifractional molecular diffusion using FRAP. This is achieved by using fluorescently labeled latex microparticles and fluorescein isothiocyanate (FITC) as probes. Multifractional diffusion of a model protein cytochrome $\mathrm{C}$ (CytC) loaded into multilayers assembled from hyaluronan
(HA) and polylysine (PLL) is also assessed using the proposed Sapproach.

\section{MATERIALS AND METHODS}

2.1. Chemicals. Dimethyl sulfoxide (DMSO, CAS 67-68-5, Fluka), fluoresceinisothiocyanate (FITC, CAS 3326-32-7, Sigma), sodium bicarbonate ( $\mathrm{NaHCO}_{3}$, CAS 144-55-8, Roth) were used as received. Three different types of latex particles were used in the presented work: Surface carboxylated fluorescent latex particles with a diameter of $0.116 \pm 0.005 \mu \mathrm{m}$ (Polysciences 16662, Ex/Em $441 \mathrm{~nm} / 486 \mathrm{~nm}, 2.5 \%$ (w/v) aqueous suspension); surface carboxylated fluorescent latex particles with a diameter of $0.042 \pm 0.007 \mu \mathrm{m}$ (Polysciences 16661, Ex/Em $441 \mathrm{~nm} / 486 \mathrm{~nm}, 2.5 \%$ (w/v) aqueous suspension); surface aminated latex particles with a diameter of $0.2 \mu \mathrm{m}$ (Micromod 01-01-202, $5.8 \times 1012$ particles $/ \mathrm{mL}, 25 \mathrm{mg}$ / $\mathrm{mL}$, solid density $1.03 \mathrm{~g} / \mathrm{cm}^{3}$, charge density $4 \mu \mathrm{mol} / \mathrm{g}$ ). Polyelectrolytes polyethylenimine (PEI, branched, $750 \mathrm{kDa}$, CAS 9002-98-6, Sigma-Aldrich), poly-L-lysine hydrobromide (PLL, 15-30 kDa, CAS 25988-63-0, Sigma-Aldrich) and sodium salt of hyaluronic acid (HA, $360 \mathrm{kDa}$, CAS 9067-32-7, Lifecore Biomedical) were used as received. TRIS (CAS 77-86-1) and $\mathrm{NaCl}$ were purchased from Sigma-Aldrich. $\mathrm{HCl}$ for $\mathrm{pH}$ equilibration was purchased from Merck.

2.2. Modification of Particles by FITC. Commercial latex particles with aminogroups exposed at the surface were modified by FITC. Primarily the dispersant of particles was changed from water to $0.1 \mathrm{M}$ carbonate buffer $\mathrm{pH} 9.0: 50 \mu \mathrm{L}$ of particle suspension was centrifuged $(12000 \mathrm{~g}, 2 \mathrm{~min})$, the supernatant discarded, pellets resuspended in $50 \mu \mathrm{L}$ of the carbonate buffer by a vortexer and in an ultrasound bath $(3 \times 10 \mathrm{~s})$.

Afterward, $19.47 \mu \mathrm{L}$ of $1 \mathrm{mg} / \mathrm{mL}$ FITC in DMSO was added to the sample to reach a nominal labeling ratio 10:1 (FITC: amino groups on the particle surface), shaken overnight at room temperature in darkness.

Afterward, unreacted FITC was removed. For that, the sample was centrifuged (12000 g, $30 \mathrm{~min}$ ), the supernatant discarded, water added to reach $50 \mu \mathrm{L}$ of final volume and resuspended with help of a vortexer and an ultrasound bath $(3 \times 10 \mathrm{~s})$. Separation of the unreacted FITC was repeated five times. Diameters of unmodified and FITC-labeled particles were determined by DLS (Zetasizer Nano ZS 633 nm, Malvern, UK). These measurements showed that the size of particles after modification remained unchanged and particles are well-stabilized in the suspension after the modification (Figure S4 in the Supporting Information).

2.3. Preparation of a Sample for FRAP Experiment. Two coverslips (\#1; $0.15 \mathrm{~mm}$ thick, Menzel, Germany) were used as top and bottom of a thin chamber. A frame of double-sided adhesive tape (3M, USA) was used to attach the coverslips together in a defined distance to produce a thin chamber. The thickness of the adhesive tape was about $0.08 \mathrm{~mm}$. Typically $4 \mu \mathrm{L}$ of a sample were trapped between the coverslips. The chamber protects samples from evaporation, flow of liquid and it permits sonication of a sample as well.

2.4. FRAP Measurements. FRAP measurements were performed by confocal laser scanning microscope (Zeiss LSM 510 meta/Axiovert 200M, Zeiss, Germany) using the $488 \mathrm{~nm}$ laser line of an Ar-ion laser or the $405 \mathrm{~nm}$ laser line of a diode laser. An objective of low numerical aperture (Zeiss LDAchroplan $20 x / 0.4$ corr) was employed in order to keep the shape of the focused laser beam close to a $z$-invariant cylindrical shape. 
A chosen region of interest (ROI) within the sample was primarily twice scanned at low laser intensity to obtain a reference image. Afterward, a rectangular area within the ROI was bleached by scanning the laser at its full power 50 or four hundred times (it typically spans a few seconds) over the area to be bleached. Afterward, the recovery process was recorded by scanning at low laser intensity over the ROI 30 or 60 times. The time between successive scans was chosen according to the expected rate of diffusion to 0.5 or $1.0 \mathrm{~s}$. Pixel scanning time was chosen as long as possible (1.6 or $3.2 \mu \mathrm{s}$, respectively) to accumulate enough signal and reduce the level of noise. Recorded images have a spatial resolution of 512 pixels $\times 512$ pixels. The dimension of ROI was adjusted by the digital zoom according to the expected rate of diffusion so that both fast and slow recovery processes are observable within the area chosen. The bleached region was 15 pixels $\times 512$ pixels large. The width of this region ( 15 pixels) provides a good compromise between a region wide enough to accumulate a sufficient amount of bleached molecules and a region narrow enough for approximating the recovery profiles by the Gauss function (the recovery profiles are of ideal Gaussian shape when bleaching of an infinitely thin region occurs instantaneously). Bleaching of a rectangular area with the long side being parallel to the scanning direction of the microscope ( $y$ axis) is the most time-efficient mode of bleaching. This bleaching mode has been employed here. The experimental setup allowed us to observe a 1D diffusion process in a limited $3 \mathrm{D}$ environment. Thanks to this spatial reduction, the amount of data to be evaluated is significantly reduced and an evaluation is faster.

2.5. FRAP Data Preprocessing. Recorded image stacks were cropped in the direction perpendicular to the direction where recovery takes place (about 128 pixels on both sides) to limit edge effects emerging during the recovery process. Afterward, the image stacks were averaged along the same dimension, which resulted in a time stack of raw FRAP profiles (ImageJ 1.51, Wayne Rasband, NIH). Please note that image lines, which were included in averaging, contain redundant information. By the averaging, a signal-to-noise ratio increases and vital information stays untouched. Resulting profiles were transferred into spreadsheet templates (Excel 2010, Microsoft) for data corrections and further analyzed.

The stack of raw FRAP profiles is primarily normalized and corrected for spatial inhomogeneities of fluorescence (postbleach profiles are divided by a master prebleach profile which was prepared by averaging ten spatiotemporally near points of prebleach profiles). Postbleach profiles are afterward corrected for unwanted bleaching which occurs during scanning and consequential edge effect (every postbleach profile is multiplied by an optimized constant to reach a value of the profile baseline close to unity). Edge effects caused by diffusion from outside the ROI may appear when unwanted bleaching was significant. The area affected by this edge effect is identified and excluded from further evaluation. A schematic overview of an image stack transformation into FRAP profiles is outlined in Figure 1.

2.6. FRAP Data Evaluation by the A-Approach. As it was described by Seiffert et al., ${ }^{19}$ FRAP recovery profiles of a single fraction can be approximated by a Gauss function, which is an analytical solution of a recovery process based on instantaneous bleaching of an infinitely thin region. Measurements should adhere to these requirements to keep evaluation by this approach reliable. Deviations from this approximation can be partially amended by an introduction of the time shift $t_{0}$ (as it will be described in eq 3 and eq 4).

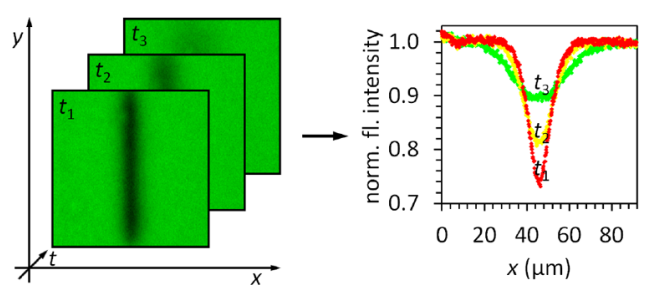

Figure 1. Schematic representation of a time stack of FRAP images recorded during the recovery process (left) and the corresponding corrected 1D FRAP profiles (right) extracted from the images.

Corrected FRAP profiles are primarily fitted by a Gauss curve defined as

$$
I(x, t)=I_{0}(t)-A(t) \cdot \exp \left(-\frac{x^{2}}{2 w^{2}}\right)
$$

where $I(x, t)$ is the fluorescence intensity at a distance $x$ from the center of the dip and at a time point after bleaching $t, I_{0}(t)$ is the fluorescence intensity of the background (ideally its value is constant over the entire ROI and close to 1$), A(t)$ is the depth of the dip at the time $t, w$ is a width of Gaussian between inflection points. All four parameters (width of the dip, spatial position of the dip, depth of the dip, intensity of the background) are free for fitting with every separate FRAP profile during Gaussian fitting. Fitting is performed as a minimization of the sum of squared deviations (SQD) of every separate measured FRAP profile from its respective Gaussian fit. After fitting a Gaussian function to every FRAP profile, the diffusion coefficient $D$ is evaluated as a half of slope of the plot $w^{2}$ versus $t$ :

$$
D=\frac{w^{2}}{2 t}
$$

This plot is linear only in case of single-fractional diffusion. When more fractions are involved in the diffusion process, this plot deviates from a linear behavior and its slope may serve only as a hint of the rate of diffusion of a major fraction. The slope at the onset of this plot rather corresponds to the recovery of fast fractions, while at later time points (when the influence of fast fractions disappears) the slope rather represents slower fractions.

The dimensionality of diffusion $d$ may also be evaluated from the slope of the plot $\log (A)$ versus $\log \left(t+t_{0}\right)$ :

$$
\log (A)=-\frac{d}{2} \cdot \log \left(t+t_{0}\right)+\text { const }
$$

where the newly introduced parameter $t_{0}$ refers to a time shift correcting deviations from the approximation of instantaneous bleaching of an infinitely thin region. The time shift can be found by varying its value until the plot $\log (A)$ versus $\log \left(t+t_{0}\right)$ become linear (here implemented as maximization of the coefficient of determination of an expected linear function). Nevertheless, because also this plot tends to deviate in a case of multifractional diffusion, typically only the first 10 points were used for evaluation of $d$. Evaluation of $D$ and $d$ from fitted Gaussians is graphically depicted in Figure 2.

The relative amount of the immobile fraction $K_{\text {rel }}$ is evaluated from the time development of the dip depth $A(t)$ by fitting the function:

$$
A(t)=\frac{M}{4 \pi D\left(t+t_{0}\right)^{d / 2}}+K
$$




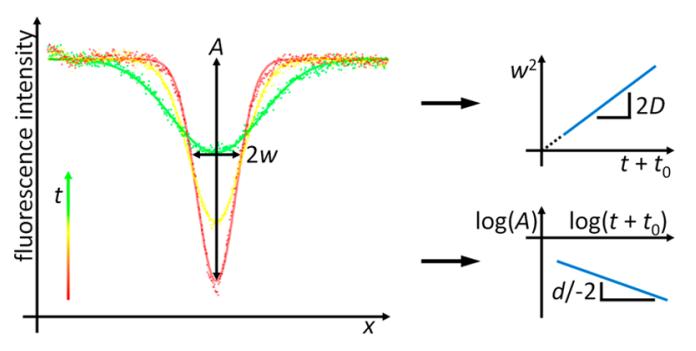

Figure 2. Schematic representation of the main steps of single-fractional analysis using the A-approach. First, Gauss functions are fitted into corrected 1D FRAP profiles (left). Second, the diffusion coefficient $D$ (right top) and the dimensionality of diffusion $d$ (right bottom) are obtained from appropriate plots of the parameters.

where $A(t)$ is the depth of the dip at time point $t, M$ is the overall reduction of fluorescence intensity caused by bleaching, $D$ is the diffusion coefficient, $t$ is time after bleaching, $t_{0}$ is correction of the time shift, $d$ is the dimensionality of diffusion, and $K$ is the partial depth of dip attributed to the immobile fraction. Values of $D$ and $d$ are taken from the previous evaluations (eq 2, eq 3 ) and fixed during the fitting. $D$ and $d$ tend to be underestimated when an amount of the immobile fraction is increased. At the same time, a presence of these values during fitting is essential for estimation of an amount of the immobile fraction. The lower limit of the $d$ value is 1 , because values lower than 1 do not reflect reality. $M, t_{0}$, and $K$ are free parameters for fitting. The input values $A(t)$ are acquired directly from the FRAP profiles, not from fitting the Gaussian function, because the evaluation of immobile fraction amount must be independent from the evaluation of $D$ as much as possible. Fitting is performed as a minimization of SQD between measured data and fitted function.

Afterward, $K_{\text {rel }}$ is obtained by relating $K$ to the depth of the dip of the first postbleach FRAP profile:

$$
K_{\mathrm{rel}}=\frac{K}{A_{0}}
$$

where $K_{\text {rel }}$ stands for the relative amount of the immobile fraction, $K$ is the depth of the dip attributed to the immobile fraction resulting from eq $4, A_{0}$ is the depth of the dip just after bleaching.

Such an approach for the evaluation of the amount of the immobile fraction requires that only two fractions are presented in the sample-a single mobile fraction with a certain $D$ and an immobile one. Otherwise, an evaluation of the amount of the immobile fraction would give only approximate results. When the amount of the immobile fraction is known, a contribution of this fraction can be subtracted from raw FRAP profiles. This step is based on an assumption, that the FRAP profile of the immobile fraction is time invariant and the shape of the immobile fraction profile is identical to the first postbleach FRAP profile. Thus, the contribution of the immobile fraction may simply be subtracted from raw FRAP profiles and the whole evaluation procedure can then be repeated. Results of this repeated evaluation allow extracting $D$ of the pure mobile fraction.

2.7. FRAP Data Evaluation by the S-Approach. 2.7.1. General Description. The S-approach consists of two consecutive steps: (1) simulation of possible single-fractional scenarios of fluorescence recovery; (2) fitting a linear combination of the single-fractional scenarios to the measured recovery profiles. The corrected postbleach FRAP profiles (as described in a Section 2.5) serve as input data for the evaluation performed by the S-approach.

Primary, the first postbleach profile is used as a starting point for the simulation of the temporal development of recovery profiles within a chosen range of diffusion coefficients. The simulation employs Ficks law for assessment of diffusion process observed. Calculations are based on the finite-difference method with a spatial mesh matching the resolution of measured data (typically hundreds of $\mathrm{nm}$ ) and a temporal mesh much finer than the repetition time of scanning during the measurement (typically $0.1 \mathrm{~ms}$ ). To further improve the numerical stability of the simulation a five-point stencil method is applied in the calculations. To suppress possible edge effect during simulation (diffusion may substantially spread out of the ROI) the spatial size of simulated profiles may be extended during simulation and afterward cropped to the original size to be saved (typically a 3fold extension of the spatial size was used). Output data of the simulation are single-fractional postbleach recovery profiles in a 2D matrix (one spatial and one temporal dimension), saved separately for every simulated diffusion coefficient (spatiotemporal dimensions of output data match the dimensions of the real measured data).

Second, the data of the real measurement are compared with possible linear combinations of simulated single-fractional recovery scenarios until the best fit (characterized by minimization of the sum of squared deviations) is found. A searching engine is based on the non-negative least-squares (NNLS) algorithm regularized by the Tikhonov-Phillips method, which is a common approach in solving inversed illposed problems. ${ }^{31}$ Solving such a problem typically requires finding a balance between the amount of meaningful information used as input for an evaluation and the amount of output information. In other words, we are searching for the simplest solution that explains most of our observed data. The need for the simplest solution is in accordance to the principle of parsimony in a meaning of Occam's razor. The employed Tikhonov-Phillips regularization penalizes more complicated solutions and thus promotes simpler solutions.

When the program presented here finds an optimal match between real measurement and linear combination of simulated data, three sets of results are obtained: FRAP profiles of a composed model, corresponding residuals and the relative amount (weight) of diffusing fractions. A schematic overview of evaluation process performed by the $S$-approach is shown in Figure 3.

All calculations of the S-approach are performed within a newly designed Java-based program with a graphical user interface (screenshots presented in Figure S1 in the Supporting Material). Main aspects of this program are universality toward evaluated data, simplicity of use and independency on the operating system used. This program is designed to evaluate unidimensional FRAP profiles for the characterization of unidimensional diffusion. It ensures simplicity and swiftness of the evaluation. Nevertheless there are no theoretical restrictions toward evaluation of data of higher dimensionality with an equivalent evaluation procedure.

2.7.2. Mathematical Apparatus. A set of experimentally measured FRAP profiles $m(x, t)$ with the distribution of diffusion coefficients $p(D)$ can be written as

$$
m(x, t)=\int p(D) c(D, x, t) d D
$$




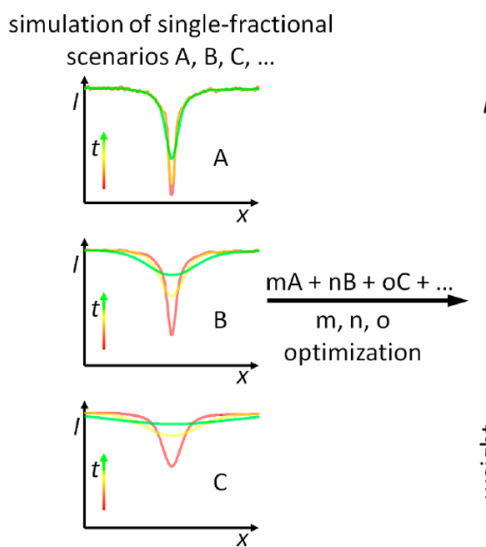

composed model fitted into data of real measurement

Figure 3. Schematic representation of the S-approach for FRAP data evaluation. Primary, possible scenarios of FRAP profile development are simulated using a certain set of diffusion coefficients (left). Second, the parameters of a linear combination of simulated single-fractional scenarios are optimized until a sufficient fit between the composed model (solid line) and measured data (points) is found (right top). Resulting optimized parameters correspond to the relative weights of diffusing fractions with corresponding diffusion coefficients $D$ (right bottom).

where $x$ denotes a spatial coordinate and $t$ the time. The term $c(D, x, t)$ describes the concentration of any component with diffusion coefficient $D$ at position $x$ and time $t$ and is given by the Fick's law:

$$
\frac{\partial c}{\partial t}=D \frac{\partial^{2} c}{\partial x^{2}}
$$

A finite difference method was utilized for iterative computation of $c(D, x, t)$ from any starting condition $c(D, x, t$ $=0)$. The concentration $c$ in time $t+\Delta t$ at position $x$ can be written:

$$
c(x, t+\Delta t)=c(x, t)+D \Delta t \operatorname{gradient}(x, t)
$$

The distribution of diffusion coefficient $p(D)$ can be approximated by a set $P(D)$ of $N$ discrete values referring to the interval of diffusion coefficients between $D_{\min }$ and $D_{\max }$. Utilizing this approximation, eq 6 can be transformed into a leastsquares problem allowing the estimation of $P(D)$ :

$$
\min \sum_{x} \sum_{t}\left[m(x, t)-\sum_{i=1}^{N} P_{i} c\left(D_{i}, x, t\right)\right]^{2}
$$

The summation is over all data points of all concentration profiles within the spatial and time interval considered for analysis. Non-negativity of the $P(D)$ coefficients was achieved by the non-negative least-squares algorithm (NNLS) from Lawson and Hanson. ${ }^{32}$

Regularization has found widespread applications, for example, for the size-distribution analysis by dynamic light scattering as well as for processing data of analytical ultracentrifugation experiments. ${ }^{31,33-35}$ Here, regularization was introduced to avoid nonrealistic oscillations in estimated set of $P$ values. In this way eq 9 was extended by adding a TikhonovPhillips regularization term, ${ }^{33}$ such that the value of integrated second derivative of the distribution $P(D)$ serves as an additional penalty in the fit optimization:

$$
\begin{aligned}
& \min \left\{\sum_{x} \sum_{t}\left[m(x, t)-\sum_{i=1}^{N} P_{i} c\left(D_{i}, x, t\right)\right]^{2}\right. \\
& \left.+\lambda \sum_{i=2}^{N-1}\left(P_{i-1}+P_{i+1}-2 P_{i}\right)^{2}\right\}
\end{aligned}
$$

From the set of all possible distributions $\{P(D)\}$ that lead to a statistically acceptable fit of the raw data, the constrain of Tikhonov-Phillips regularization based on the second derivative term reflects our assumption that the "true" distribution of diffusion coefficients has no sharp peaks. The regularization penalty leads to an increase in the variance $\sigma$ of the fit with an increasing magnitude of the regularization parameter $\lambda$. The relative variance increase $F=\sigma(\lambda) / \sigma(\lambda=0)$ follows a Fisher distribution and can be exploited to adjust the magnitude of the regularization parameter such that the quality of the regularized fit still remains statistically indistinguishable from the unconstrained best fit on a given confidence level. ${ }^{35,36}$ Because of a large number of data points, the effect of the constraint on the number of degrees of freedom can be neglected.

2.8. HA/PLL Multilayers Loaded with CytC. The PEI$(\mathrm{HA} / \mathrm{PLL})_{23} \mathrm{HA}$ polyelectrolyte multilayer deposited on a glass fiber $(0.1 \mathrm{~mm}$ in diameter, $1 \mathrm{~cm}$ long) was prepared by the layerby-layer assembly. Glass fiber (cleaned by $2 \%$ Hellmanex III, followed by $1 \mathrm{M} \mathrm{HCl}$ and water) was sequentially incubated in solutions of appropriate polyelectrolytes with intermediate washing steps using an automated dipping robot (DR3, Riegler \& Kirstein) until the PEI(HA/PLL $)_{23} \mathrm{HA}$ composition was achieved. Each incubation of the substrate in $0.5 \mathrm{mg} / \mathrm{mL}$ solution of appropriate polyelectrolyte lasted $10 \mathrm{~min}$. The intermediate washing in buffer consisted of three steps lasting for $3 \mathrm{~min}$ each. The multilayer assembly and further experiments with multilayers were performed in $10 \mathrm{mM}$ TRIS buffer of $\mathrm{pH} 7.4$ supplemented with $15 \mathrm{mM} \mathrm{NaCl}$. The multilayers were loaded with FITC labeled CytC by incubating them in about $50 \mu \mathrm{L}$ of 30 $\mu \mathrm{M}$ buffer solution of FITC labeled CytC for an hour at RT. Afterward, the fiber was washed three times with the buffer and further kept in $100 \mu \mathrm{L}$ of the buffer for series of FRAP experiments performed at progressing time after loading.

\section{RESULTS AND DISCUSSION}

3.1. Evaluation of a Single-Fractional FRAP Data. A sample composed of a single fraction of microparticles that is characterized by one diffusion coefficient was utilized in a first step for the validation of the S-approach for evaluating FRAP data. The sample consists of a stable aqueous suspension of fluorescent latex microparticles of 0.116 or $0.042 \mu \mathrm{m}$ diameter. These particles should diffuse with diffusion coefficients of 4.2 or $12 \mu \mathrm{m}^{2} / \mathrm{s}$ respectively according to the Stokes-Einstein equation (at $25{ }^{\circ} \mathrm{C}$ ). Moreover the samples were prepared at several dilutions $(1,2,4,8$ times dilution) to assess the influence of interactions between particles (restricted diffusion) and a range of particle concentration applicable for the measurement. The data obtained were evaluated first by the A-approach and afterward by the S-approach. Data presented in this section are based on 30 images recorded during 15 seconds of the recovery process.

3.1.1. A-Approach. Expectations of the evaluation procedure on the evaluated data are straightforward. But the results of the analysis become easy misleading when data does not fulfill some of the expectations (e.g., deviations from expected shape of 
Table 1. Results of Evaluation of Data from FRAP Experiment with a Single Diffusive Fraction by the A-Approach Using 0.116 or $0.042 \mu \mathrm{m}$ Sized Particles as Probes ${ }^{a}$

\begin{tabular}{|c|c|c|c|c|c|c|c|c|}
\hline particle diameter $(\mu \mathrm{m})$ & \multicolumn{4}{|c|}{0.116} & \multicolumn{4}{|c|}{0.042} \\
\hline particle dilution factor & 1 & 2 & 4 & 8 & 1 & 2 & 4 & 8 \\
\hline$D\left(\mu \mathrm{m}^{2} / \mathrm{s}\right)^{b}$ & $4.7 \pm 0.4$ & $4.6 \pm 0.3$ & $5.0 \pm 0.3$ & $4.8 \pm 0.8$ & $14.5 \pm 0.4$ & $14.8 \pm 0.7$ & $15.4 \pm 0.8$ & $15.3 \pm 0.7$ \\
\hline$d^{c}$ & $0.90 \pm 0.09$ & $0.87 \pm 0.07$ & $1.07 \pm 0.26$ & $0.80 \pm 0.25$ & $0.99 \pm 0.07$ & $0.99 \pm 0.03$ & $0.93 \pm 0.04$ & $0.93 \pm 0.09$ \\
\hline amount of immobile fraction (\%) & $1 \pm 2$ & $1 \pm 2$ & $2 \pm 3$ & $5 \pm 4$ & $0 \pm 0$ & $0 \pm 0$ & $0 \pm 0$ & $0 \pm 0$ \\
\hline$M\left(\mu \mathrm{m}^{d}\right)^{d}$ & $2.7 \pm 0.2$ & $3.4 \pm 0.1$ & $4.6 \pm 0.5$ & $4.7 \pm 0.2$ & $3.5 \pm 0.3$ & $4.2 \pm 0.3$ & $4.4 \pm 0.5$ & $6.0 \pm 0.3$ \\
\hline $\mathrm{SQD}^{e}$ & $0.34 \pm 0.03$ & $0.43 \pm 0.01$ & $1.07 \pm 0.12$ & $2.51 \pm 0.23$ & $0.20 \pm 0.04$ & $0.35 \pm 0.02$ & $0.81 \pm 0.08$ & $2.29 \pm 0.11$ \\
\hline
\end{tabular}

${ }^{a}$ Displayed numbers represent mean \pm standard deviation (based on triplicates). ${ }^{b} D$ was evaluated from initial $2.5 \mathrm{~s}$ of recovery. ${ }^{c} d$ was evaluated from the initial $5 \mathrm{~s}$ of recovery. ${ }^{d} \mathrm{M}$ quantifies the fluorescence dip produced during pattern bleaching. ${ }^{e} \mathrm{SQD}$ correspond to the difference between all 30 measured FRAP profiles and their respective Gaussian fits.

bleached profile, substantially long bleaching procedure, multifractional diffusion). As it was described in the Section 2.6 a full data evaluation requires three separate fitting steps.

First, Gaussians are fitted to FRAP profiles and corresponding diffusion coefficient can be directly evaluated as described in eq 1 and 2 . The fitting procedure is typically able to identify a correct fit of the Gaussians on a first run. Occasionally, the fitting procedure may fail at later time points of the recovery process, when the depth of an observed dip becomes smaller than the noise level and/or diffusion spreads the dip substantially behind ROI. Nevertheless, just several correctly fitted FRAP profiles from the onset of recovery are typically enough for the determination of $D$.

Second, the $t_{0}$ parameter is varied in an iterative process until the graph characterized by eq 3 becomes linear. This graph is fairly susceptible to irregularities introduced from real measurement data arising from instability of offset or sensitivity of the detector and other variables. Values at later time points of recovery are often unstable and thus only early stages of recovery with a uniform trend are suitable for the optimization of $t_{0}$. Even a careful choice of the time interval included into the optimization of $t_{0}$ does not necessarily result in robust value of dimensionality of diffusion $d$.

Third, eq 4 is fitted to the measured time evolution of the depth of the $\operatorname{dip} A(t)$. Parameters $D$ and $d$ of eq 4 are acquired from the previous steps of evaluation, while parameters $t_{0}, K$, and $M$ are free for fitting. Results of this evaluation are also fairly susceptible to irregularities introduced from measured data. Moreover, the result does not necessarily possess only one welldefined minima of SQD but may contain several minima in case of noisy input data or poorly shaped recovery profiles. Thus, the fitting procedure may identify a solution with local minima of SQD, which does not represent reality. Robustness of the evaluated amount of immobile fraction is thus strongly dependent on the quality of input data.

Results of fitting presented here are based on optimal but real experimental conditions and thus should represent the best possible outcome. The results are summarized in Table 1. From the table it can be concluded that results of the A-approach match expected values and trends. Importantly, the obtained $D$ of the microparticles does not vary with changing dilution factor and match expected values of 4.2 or $12 \mu \mathrm{m}^{2} / \mathrm{s}$ for 0.116 and $0.042 \mu \mathrm{m}$ sized particles, respectively. It indicates no influence of interactions between particles and also no effect of the overall intensity of fluorescence signal within the chosen dilution range on the resulting $D$. The evaluated dimensionality of diffusion $d$ is close to unity. It confirms that the setting of the experiment (low NA objective and bleaching of prolonged rectangular area) effectively reduces dimensionality of the observed diffusion to $1 \mathrm{D}$ in a restricted 3D space. An effect of NA of the objectives used is presented in Table S1. The evaluated amount of immobile fraction is negligible or zero in all samples. This result fulfills our expectations for pure single-fractional diffusion. Further results indicate that the amount of bleached fluorophores $M$ increases with an increasing dilution factor. We have not found a satisfactory explanation for this observation until now. Our measurements indicate, that this observation cannot be fully explained by depletion of excitation photons due to absorption in the sample neither by scattering of the excitation light by latex particles. The resulting SQD after Gaussian fitting increases with an increase of the dilution factor. It corresponds to decrease of fluorescence signal while the noise of recorded profiles stays unchanged.

3.1.2. S-Approach. The S-approach allows us to evaluate multifractional diffusion without any precedent information about a number of fractions. Both single-fractional and multifractional diffusion can be assessed. In contrast to the Aapproach, no particular shape of the recovery profile is required - FRAP profiles are evaluated as they are. The S-approach evaluates a whole set of data from FRAP experiment at once, unlike the analytical solution where evaluation proceeds in a stepwise manner. This freedom makes data evaluation more potent but it also requires more careful assessment of uncertainty of the results.

Results of the evaluation by the S-approach are documented in Figure 4 and are also summarized in Table 2. Diffusion coefficients obtained by the S-approach are in a good agreement with expected values. According to the Stokes-Einstein equation, the expected diffusion coefficients for the 0.116 and $0.042 \mu \mathrm{m}$ sized microparticles are 4.2 and $12 \mu \mathrm{m}^{2} / \mathrm{s}$, respectively. The results are also in line with results obtained by the analysis of the identical data by the A-approach (Table 1). This proves the ability of the S-approach to provide correct results.

The regularization parameter $\lambda$ was set to 10 for all evaluations. This value was chosen just high enough to keep evaluated distributions of the diffusion coefficient presented in this section in a form of a single compact peak. An optimal value of $\lambda$ depends on the signal-to-noise $(\mathrm{S} / \mathrm{N})$ ratio of the input data and on the amount of input data. The first mentioned $\mathrm{S} / \mathrm{N}$ ratio is related to the quality of the input data and it typically falls into the interval from 30 to 50 in experiments presented in Figure 4. (The S/N was calculated as the ratio between the depth of the dip of the first postbleach profile and the standard deviation of the noise of the FRAP profile.) The second mentioned amount of input data is practically an amount of well-shaped FRAP profiles. Various experiments will differ in both parameters but variations within here presented experiments are not significant. A value $\lambda$ equal to 10 is a good approximation for most of the cases presented here 

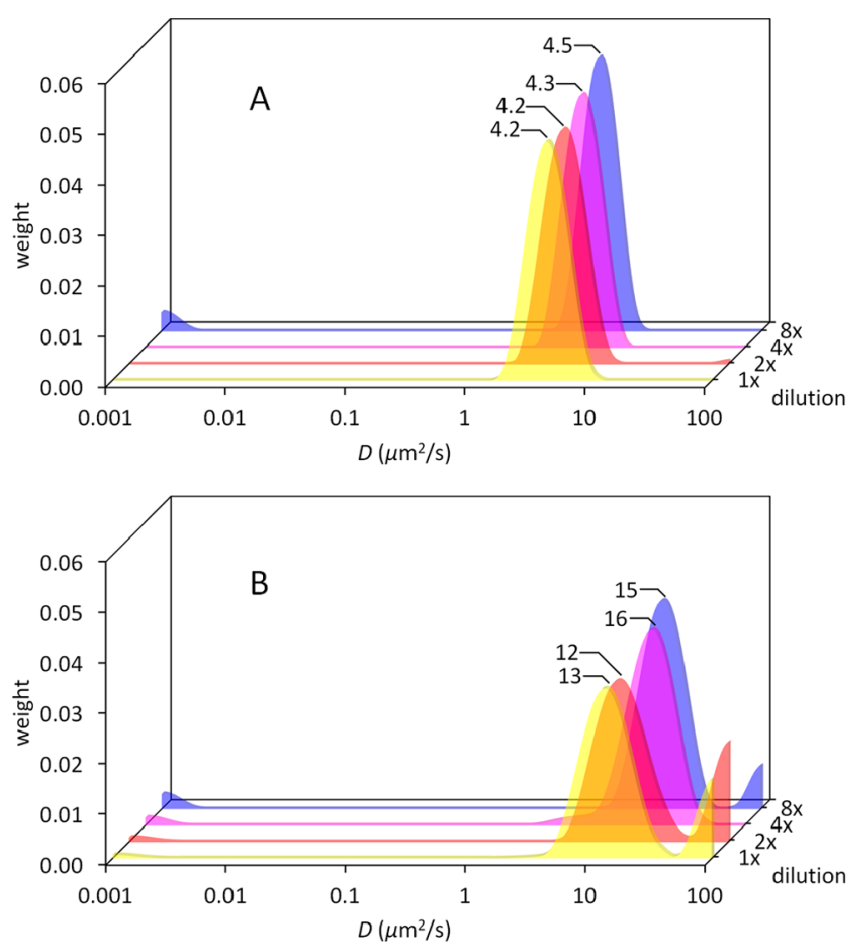

Figure 4. Distribution of diffusion coefficients obtained by the $S$ approach for 0.116 (A) and $0.042 \mu \mathrm{m}$ (B) sized particles, respectively. The presented distributions are averaged distributions form triplicate measurements. Values denoted at peaks are diffusion coefficients at peak maxima. Regularization parameter $\lambda=10$.

and keeping this value fixed simplifies comparison between different experimental results. More results on the use of the regularization can be found in the Section 3.3.

3.2. Evaluation of a Two-Fractional FRAP Data by the SApproach. A sample consisting of two components of different diffusivity was used to test the ability of the S-approach to resolve two differently diffusive particle fractions. The slow diffusing component was a stable suspension of FITC-conjugated latex microparticles $(0.2 \mu \mathrm{m}$ in diameter $)$ and the fast diffusing component was hydrolyzed FITC. The FITC conjugated particles and hydrolyzed FITC were exploited for this experiment to ensure comparable fluorophore behavior of both components. A ratio between these two components within the sample was varied. Data presented in this section are based on 60 images recorded during 60 seconds of the recovery process.

The results presented in Figure 5 demonstrate the ability of the evaluation procedure to resolve two diffusive fractions within one sample and to identify the diffusion coefficients of the fractions. $D$ of $0.2 \mu \mathrm{m}$ sized microparticles is approximately $2.2 \mu \mathrm{m}^{2} / \mathrm{s}$ according to DLS measurements, and D of fluorescein (or

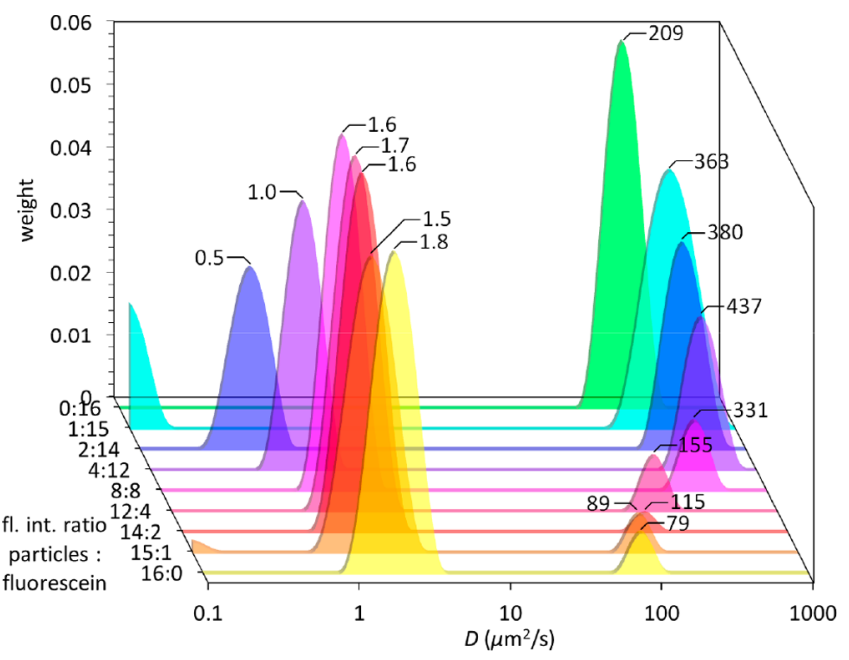

Figure 5. Distribution of diffusion coefficients of samples with two diffusive fractions $(0.2 \mu \mathrm{m}$ sized particles and FITC) obtained by the evaluation of FRAP experiments using the S-approach. The fractions were mixed at defined ratios corresponding to their fluorescence intensity. Values denoted at peaks are diffusion coefficients at peak maxima. The diffusion coefficient of particles is $2.2 \mu \mathrm{m}^{2} / \mathrm{s}$ as determined by DLS measurement and the diffusion coefficient of hydrolyzed FITC is given to be approximately $420 \mu \mathrm{m}^{2} / \mathrm{s} .{ }^{37}$ Regularization parameter $\lambda=$ 10.

hydrolyzed FITC, respectively) is given to be approximately 420 $\mu \mathrm{m}^{2} / \mathrm{s}$.

The results of the evaluation by the $S$-approach are displayed in Figure 5. The $D$ values of FITC decrease from 437 to 209 $\mu \mathrm{m}^{2} / \mathrm{s}$ with increasing FITC concentrations from particle:FITC ratios of $4: 12$ to $0: 16$. Fluorescein is known to form dimers at higher concentrations, which would alter its diffusion speed. Nevertheless, just about $0.025 \%$ of fluorescein should be present in dimeric form at its highest concentration $(25 \mu \mathrm{M})$ used in our experiments (dimerization constant of fluorescein dianion is about $\left.5 \mathrm{M}^{-1}\right) .{ }^{38,39}$ The actual amount of dimers may differ because of a shift of the dimerization constant caused by differences in ionic strength, $\mathrm{pH}$ and because the behavior of FITC may differ from fluorescein (Figure S6). This dimerization phenomenon could partially explain the observed down-shift of $D$ of FITC but probably additional effects are involved.

$D$ of FITC fractions at high particle:FITC ratios (over 12:4) readily drop to values of approximately $100 \mu \mathrm{m}^{2} / \mathrm{s}$. Surprisingly, the peak of this fraction is present even in case, when no FITC was added (a "16:0" ratio of Figure 5). The origin of the fraction corresponding to this peak is not clearly known. Nevertheless results indicate that this peak may correspond to FITC residuals being still present after the coupling reaction or to fluorophore

Table 2. Results of Evaluation of Data from FRAP Experiments with a Single Diffusive Fraction by the S-Approach Using 0.116 or $0.042 \mu \mathrm{m}$ Sized Particles as Probes ${ }^{a}$

\begin{tabular}{|c|c|c|c|c|c|c|c|c|}
\hline particle diameter $(\mu \mathrm{m})$ & & & & & & & & \\
\hline particle dilution factor & 1 & 2 & 4 & 8 & 1 & 2 & 4 & 8 \\
\hline$D\left(\mu \mathrm{m}^{2} / \mathrm{s}\right)^{b}$ & $4.2 \pm 0.1$ & $4.2 \pm 0.1$ & $4.3 \pm 0.1$ & $4.5 \pm 0.1$ & $13 \pm 2$ & $12 \pm 2$ & $16 \pm 2$ & $15 \pm 1$ \\
\hline
\end{tabular}

${ }^{a}$ Displayed numbers represent mean value \pm standard deviation (based on triplicates). ${ }^{b} D$ was evaluated as peak maximum of the distribution of diffusion coefficients resulting from S-approach analysis. ${ }^{c} \mathrm{SQD}$ corresponds to the difference between all 29 FRAP profiles and their corresponding fits found by the S-approach. 
attached to some molecular fragments released from the particle surface after the coupling reaction.

$D$ of latex particles steadily stays at a value of approximately 1.7 $\mu \mathrm{m}^{2} / \mathrm{s}$ for most mixing ratios. It agrees well with the value 2.2 $\mu \mathrm{m}^{2} / \mathrm{s}$, which corresponds to $D$ determined by DLS. The position of this peak starts to shift to lower values, when the fluorescence signal of this fraction becomes significantly weaker as mixing ratio drops. This shift may be caused by an artifact of the data evaluation originating from the assumption that the bleaching procedure is much faster than the observed diffusion and the first postbleach FRAP profile is recorded immediately after bleaching. Because this assumption is not completely fulfilled, the first postbleach profile is not the true starting point of diffusion for all fractions, but recovery through diffusion of particles is already underway at this stage. As the recovery process takes place, the fast fractions recover swiftly. The slow fraction on the contrary still forms a well-defined dip in the FRAP profile, but in comparison with the first postbleach profile it appears narrower than expected, because mostly the fast fraction caused broadening of FRAP profile in the first postbleach image. Thus, the diffusion speed of the slow fraction is underestimated, when the fast fraction dominates.

A sample consisting of pure hydrolyzed FITC (a "0:16" ratio of Figure 5) shows only a single peak as it is expected but its position is somehow down-shifted (partially explainable by dimerization of fluorescein as described above).

The distributions from Figure 5 are of a peak width of a quarter decade of $D$ approximately. It can serve as an indication of resolving power of the $S$-approach applied to this particular set of data with the regularization parameter $\lambda$ set to be 10 . Because the regularization procedure generally tends to merge overlapping peaks (details in Figure S5), the distance between two peaks should be at least about half a decade ( $\sim$ three times) of $D$ in order to be resolved. The $\mathrm{S} / \mathrm{N}$ ratio of input data of Figure 5 typically falls into an interval between 40 and 110 .

A mixture of monomeric and dimeric FITC molecules, as well as a mixture of latex particles ( 0.1 and $0.5 \mu \mathrm{m}$ in diameter), have been assessed for multifractional analysis by $\mathrm{S}$-approach as well (Figures S2 and S3, respectively). Unfortunately, it was not possible to resolve two distinct fractions in either case. This may be explained by an insufficient $\mathrm{S} / \mathrm{N}$ ratio of input data.

3.3. Regularization and Limitations of the S-Approach. The S-approach uses a regularization of possible solutions during the optimization phase to balance the amount of information on the output to the amount and quality of information on the input. The better the quality and the larger the quantity of the input data, the weaker the regularization may be applied and thus the higher the resolving power becomes. We have observed that the quality of our data was mostly hampered by noise of the detector (photomultiplier tube used in the CLSM). Better S/N ratios could be possibly obtained with better detectors. The quality of input data for multifractional analysis was negatively influenced also by deviations from an assumption of instantaneous bleaching. This issue can be reduced by the combination of installing a high power laser module and employing a fluorophore prone to bleaching in order to reduce the bleaching time. To avoid this problem at all, the bleaching process could be integrated as a part of a simulation-based evaluation. We have decided not to include a simulation of the bleaching process into our S-approach because it would require the introduction of additional parameters describing the bleaching process to be optimized or known in advance. ${ }^{40,41}$
The number of recorded well-shaped FRAP profiles defines the input data quantity. The slower the observed components diffuse, the more FRAP profiles can be recorded. When a fast recovery is observed (like recovery of molecular hydrolyzed FITC) it may take only several seconds until a bleached pattern disappears in the noise. Here, we use a scanning rate of two images per second or one image per second. Higher scanning rates would not improve the results any further, because it would lead to a significant increase of noise of FRAP profiles recorded with our CLSM setup.

The program (NBJ-FRAP-1) presented in this study employs the Tikhonov-Phillips method of regularization. This regularization method penalizes solutions with higher values of the second derivatives of the distribution of diffusion coefficients. Strength of the regularization may be varied by adjusting the parameter $\lambda$. When the strength of regularization is chosen too weak, the evaluation procedure may generate a distribution that indicates additional diffusive fractions presented around position of an expected single diffusive fraction-peak decomposition takes place. When a higher strength of regularization is chosen, peaks in the distribution tend to become broader and smoother. When two peaks are close enough, they tend to merge into a single peak, the decomposed peaks merge. This phenomenon is documented in Figure S5.

One of important aspects of simulation-optimization methods is the computational burden. A weight of the burden is ruled by the chosen parameters of simulation and optimization. The simulation procedure of a typical simulation-based evaluation presented in this study spans minutes up to tens of minutes with a standard up-to-date PC. The subsequent optimization procedure (NNLS algorithm) typically requires just minutes in order to find a solution.

Some critical factors inherent to the FRAP method itself are listed with regard to the S-approach: low signal-to-noise ratio, the bleaching time comparably long as the time of recovery, the nonlinear correlation between fluorophore concentration and fluorescence intensity, fluorescence recovery through chemical reactions or any other recovery mechanisms different from the spontaneous diffusion, photoactivated side-reactions, competition between diffusion and binding reaction, local heating of the sample caused by scanning or the bleaching procedure and more. These factors may be present to a certain extent in most of the FRAP experiments. However, their influence should be minimized to keep the data evaluations valid or they should be included into the evaluation process.

3.4. Multifractional Analysis of CytC Diffusion in HA/ PLL Multilayers. The final step of this work deals with the evaluation of multifractional diffusion of a biomacromolecule (protein) loaded into a polyelectrolyte multilayer. FITC labeled CytC and PEI(HA/PLL) ${ }_{23} \mathrm{HA}$ have been chosen as a model protein and a well-studied biologically relevant multilayer, respectively. The multilayer is a few micrometers thick. Figure 6 presents the time evolution of the distribution of diffusion coefficients of CytC loaded into the multilayer. During storage in the buffer, the fast diffusing fractions of CytC diminish after some time and the slow diffusing fractions start to dominate. These results are in a good agreement with our previous findings ${ }^{42}$ demonstrating that a distribution of diffusive fractions of the protein lysozyme loaded into a similar HA/PLL multilayer changes in a comparable manner. This phenomenon may be explained by gradually increasing interactions between protein molecules or between protein and polymer(s) during the time course of storage. The observed change is rather slow probably 


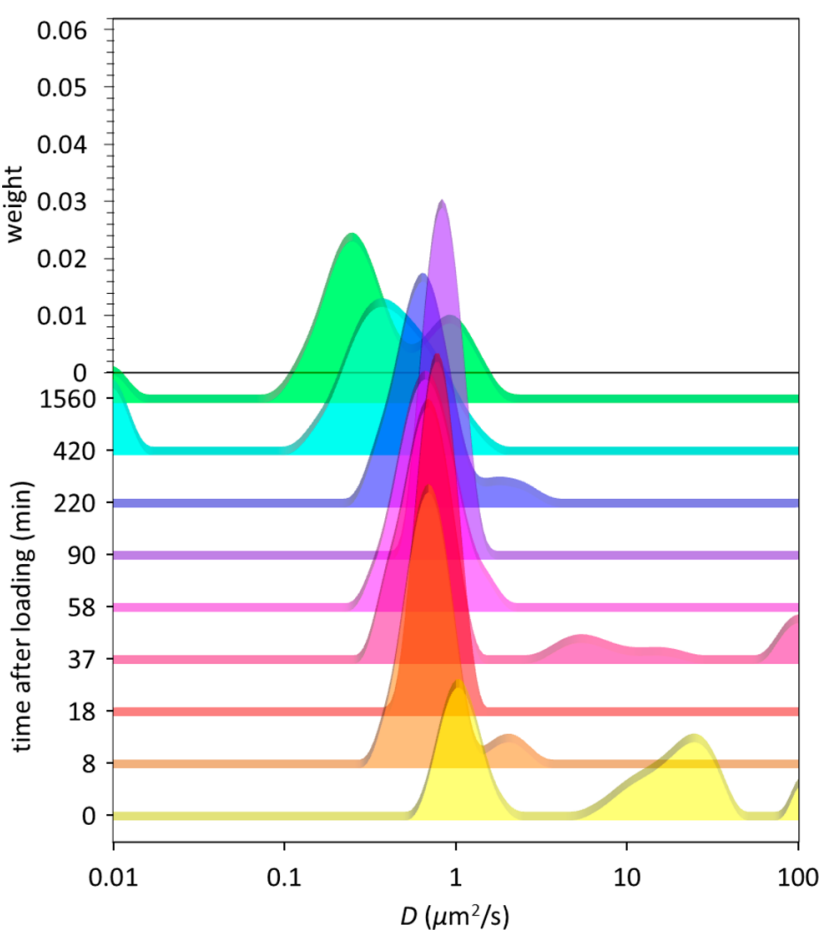

Figure 6. Distribution of diffusion coefficients of CytC loaded into (HA/PLL $)_{24}$ multilayers.

due to multiple cooperative interactions in the multilayers. We believe that the developed approach will be useful for better understanding of diffusion phenomenon in the multilayers and its effect on the multilayer structure and properties. ${ }^{43,44}$ This, in turn, is a key to assemble biologically active multilayers with adjusted composition and tailor-made properties as a platform to guide cellular behavior. ${ }^{45-49}$

\section{CONCLUSIONS}

In this study, we have developed a novel simulationoptimization-based approach (S-approach) for the evaluation of FRAP data. This powerful tool allows evaluation of single- as well as multifractional diffusion. The approach is based on simulation of possible scenarios of the recovery process followed by fitting a linear combination of the simulated recovery profiles into the recovery profiles obtained experimentally. We have compared the classical approach based on the analytical solution of molecular diffusion (A-approach) with the S-approach by processing the same FRAP data for microparticles. Results of these two approaches are well comparable when samples consist of a single diffusive fraction. In this case, both methods produce robust results. The ability of the $S$-approach to evaluate multifractional diffusion was examined on a sample consisting of two fractions of diffusion coefficients that differed by a factor of 100. The evaluation procedure was able to identify individual fractions and determine their diffusion coefficients with acceptable accuracy. However, the resolving power of the S-approach was insufficient for resolving two diffusive fractions of similar diffusivity (difference of diffusion coefficient by a factor of five or two). The achievable resolving power of the simulation-based method is governed by the quality and the quantity of the input data. To reflect this relation, the Tikhonov-Phillips regularization of possible solutions is employed. As an example for applying the S-approach, we assess the diffusion of the protein cytochrome $\mathrm{c}$ in polyelectrolyte multilayers (e.g., biopolymer- based HA/PLL films). It is shown, that the distribution of the diffusion coefficients of the protein in a polyelectrolyte multilayer shifts toward slower fractions with progressing time. This demonstrates the potential of our approach for the comprehensive analysis of modern drug delivery systems such as multilayers. It may open new avenues for controlling the presentation of biologically relevant molecules from multilayers to cells. A newly developed program (NBJ-FRAP-1) employing the $S$-approach is a user-friendly tool combining several features for the evaluation of FRAP data. It only requires the computational power of standard PCs for the swift and straightforward evaluation of the FRAP data.

\section{ASSOCIATED CONTENT}

\section{Supporting Information}

The Supporting Information is available free of charge on the ACS Publications website at DOI: 10.1021/acs.jpcb.7b11051.

Screenshots of the NBJ-FRAP-1 program, multifractional evaluation of further experiments by the S-approach, DLS analysis of latex amino-particles before and after conjugation with FITC, comparison of the observed dimensionality of diffusion during FRAP experiments employing objectives of 0.4 and 1.4 NA, an effect of the regularization parameter $\lambda$ on the solutions found by the $S$ approach, and structures of fluorescein and hydrolyzed FITC molecules (PDF)

The NBJ-FRAP-1 program (free software under GNU GPL ver. 3 or higher), source code of the program and documentation of the program (ZIP)

\section{AUTHOR INFORMATION}

\section{Corresponding Author}

*Phone: +44-115-848-3140; E-mail: dmitry.volodkin@ntu.ac. uk.

\section{ORCID}

Dmitry Volodkin: 0000-0001-7474-5329

\section{Notes}

The authors declare no competing financial interest.

\section{ACKNOWLEDGMENTS}

D.V. thanks the Alexander von Humboldt Foundation in the framework of the Sofja Kovalevskaja program, DFG grant VO 1716/2-3, and Materials Research Seed Corn Fund 2016-2017 from NTU for support. A.H. thanks the Grant Agency of the Czech Republic for financial support (Grant Number P20612G014). Natalia Velk is gratefully acknowledged for preparation of FITC labelled CytC.

\section{REFERENCES}

(1) Axelrod, D.; Koppel, D. E.; Schlessinger, J.; Elson, E. L.; Webb, W. W. Mobility Measurement by Analysis of Fluorescence Photobleaching Recovery Kinetics. Biophys. J. 1976, 16, 1055-1069.

(2) Elson, E. L.; Magde, D. Fluorescence Correlation Spectroscopy. Biopolymers 1974, 13, 1-27.

(3) Saxton, M. J. Single-Particle Tracking: The Distribution of Diffusion Coefficients. Biophys. J. 1997, 72, 1744-1753.

(4) Ernst, D.; Köhler, J. Measuring a Diffusion Coefficient by Singleparticle Tracking: Statistical Analysis of Experimental Mean Squared Displacement Curves. Phys. Chem. Chem. Phys. 2013, 15, 845-849.

(5) Müller, P.; Schwille, P.; Weidemann, T. PyCorrFit-Generic Data Evaluation for Fluorescence Correlation Spectroscopy. Bioinformatics 2014, 30, 2532-2533. 
(6) Steger, K.; Bollmann, S.; Noé, F.; Doose, S. Systematic Evaluation of Fluorescence Correlation Spectroscopy Data Analysis on the Nanosecond Time Scale. Phys. Chem. Chem. Phys. 2013, 15, 1043510445 .

(7) Hallen, M.; Layton, A. T. Expanding the Scope of Quantitative FRAP Analysis. J. Theor. Biol. 2010, 262, 295-305.

(8) Weiss, M. Challenges and Artifacts in Quantitative Photobleaching Experiments. Traffic 2004, 5, 662-671.

(9) Soumpasis, D. Theoretical Analysis of Fluorescence Photobleaching Recovery Experiments. Biophys. J. 1983, 41, 95-97.

(10) Frank, G. A.; Marconi, M. C.; Corti, H. R. An Alternate Solution of the Fluorescence Recovery Kinetics after Spot-Bleaching for Measuring Diffusion Coefficients. J. Solution Chem. 2008, 37, 15751591.

(11) Braeckmans, K.; Peeters, L.; Sanders, N. N.; De Smedt, S. C.; Demeester, J. Three-Dimensional Fluorescence Recovery after Photobleaching with the Confocal Scanning Laser Microscope. Biophys. J. 2003, 85, 2240-2252.

(12) Gordon, G. W.; Chazotte, B.; Wang, X. F.; Herman, B. Analysis of Simulated and Experimental Fluorescence Recovery After Photobleaching. Data for Two Diffusing Components. Biophys. J. 1995, 68, 766-778.

(13) Mai, J.; Trump, S.; Lehmann, I.; Attinger, S. Parameter Importance in FRAP Acquisition and Analysis: A Simulation Approach. Biophys. J. 2013, 104, 2089-2097.

(14) Sullivan, K. D.; Majewska, A. K.; Brown, E. B. Single- and TwoPhoton Fluorescence Recovery after Photobleaching. Cold Spring Harbor Protocols 2015, 2015, 13-23.

(15) Cinquemani, E.; Roukos, V.; Lygerou, Z.; Lygeros, J. Numerical Analysis of FRAP Experiments for DNA Replication and Repair. Proceedings of the 47th IEEE Conference on Decision and Control; Cancun, Mexico, December 9-11, 2008.

(16) Jonasson, J.; Hagman, J.; Lorén, N.; Bernin, D.; Nyden, M.; Rudemo, M. Pixel-based Analysis of FRAP Data with a General Initial Bleaching Profile. J. Microsc. 2010, 239, 142-153.

(17) Uhlig, K.; Madaboosi, N.; Schmidt, S.; Jäger, M. S.; Rose, J.; Duschl, C.; Volodkin, D. V. 3d Localization and Diffusion of Proteins in Polyelectrolyte Multilayers. Soft Matter 2012, 8, 11786-11789.

(18) Picart, C.; Mutterer, J.; Arntz, Y.; Voegel, J.-C.; Schaaf, P.; Senger, B. Application of Fluorescence Recovery After Photobleaching to Diffusion of a Polyelectrolyte in a Multilayer Film. Microsc. Res. Tech. 2005, 66, 43-57.

(19) Seiffert, S.; Oppermann, W. Systematic Evaluation of FRAP Experiments Performed in a Confocal Laser Scanning Microscope. J. Microsc. 2005, 220, 20-30.

(20) Tannert, A.; Tannert, S.; Burgold, S.; Schaefer, M. Convolutionbased One and Two Component FRAP Analysis: Theory and Application. Eur. Biophys. J. 2009, 38, 649-661.

(21) Jönsson, P.; Jonsson, M. P.; Tegenfeldt, J. O.; Höök, F. A Method Improving the Accuracy of Fluorescence Recovery after Photobleaching Analysis. Biophys. J. 2008, 95, 5334-5348.

(22) Hauser, G. I.; Seiffert, S.; Oppermann, W. Systematic Evaluation of FRAP Experiments Performed in a Confocal Laser Scanning Microscope - Part II: Multiple Diffusion Processes. J. Microsc. 2008, 230, 353-362.

(23) Vogt, C.; Ball, V.; Mutterer, J.; Schaaf, P.; Voegel, J.-C.; Senger, B.; Lavalle, P. Mobility of Proteins in Highly Hydrated Polyelectrolyte Multilayer Films. J. Phys. Chem. B 2012, 116, 5269-5278.

(24) Glotsos, D.; Kostopoulos, S. A.; Ninos, K.; Cavouras, D. Interpretation of Binding Kinetics in Fluorescence Recovery after Photobleaching Experiments Using a Novel Stochastic Simulation Strategy. Mol. Simul. 2009, 35, 1209-1214.

(25) Kubitscheck, U.; Wedekind, P.; Peters, R. Three-dimensional Diffusion Measurements by Scanning Microphotolysis. J. Microsc. 1998, 192, 126-138.

(26) Irrechukwu, O. N.; Levenston, M. E. Improvement Estimation of Solute Diffusivity Through Numerical Analysis of FRAP Experiments. Cell. Mol. Bioeng. 2009, 2, 104-117.
(27) Alevra, M.; Schwartz, P.; Schild, D. Direct Measurement of Diffusion in Olfactory Cilia Using a Modified FRAP Approach. PLoS One 2012, 7, e39628.

(28) Vinnakota, K. C.; Mitchell, D. A.; Deschenes, R. J.; Wakatsuki, T.; Beard, D. Analysis of the Diffusion of Ras2 in Saccharomyces cerevisiae using Fluorescence Recovery after Photobleaching. Phys. Biol. 2010, 7, 026011.

(29) Zadeh, K. S. A Synergic Simulation-optimization Approach for Analyzing Biomolecular Dynamics in Living Organisms. Comput. Biol. Med. 2011, 41, 24-36.

(30) Zadeh, K. S.; Montas, H. J.; Shirmohammadi, A. Identification of Biomolecule Mass Transport and Binding Rate Parameters in Living Cells by Inverse Modeling. Theor. Biol. Med. Modell. 2006, 3, 36.

(31) Provencher, S. W. A. Constrained Regularization Method for Inverting Data Represented by Linear Algebraic or Integral Equations. Comput. Phys. Commun. 1982, 27, 213-227.

(32) Lawson, C. L.; Hanson, R. J. Solving Least Squares Problems; Prentice-Hall: Englewood Cliffs, 1974.

(33) Phillips, D. L. A. Technique for Numerical Solution of Certain Integral Equations of First Kind. J. Assoc. Comput. Mach. 1962, 9, 84-97.

(34) Schuck, P.; Rossmanith, P. Determination of the Sedimentation Coefficient Distribution by Least-squares Boundary Modeling. Biopolymers 2000, 54, 328-341.

(35) Johnson, M. L.; Straume, M. Comments on the Analysis of Sedimentation Equilibrium Experiments. In Modern Analytical Ultracentrifugation; Schuster, T. M., Laue, T. M., Eds.; Birkhäuser: Boston, 1994; pp 37-65.

(36) Bevington, P.; Robinson, D. K. Data Reduction and Error Analysis for the Physical Sciences; McGraw-Hill: Boston, 1992.

(37) Culbertson, C. T.; Jacobson, S. C.; Ramsey, J. M. Diffusion Coefficient Measurements in Microfluidic Devices. Talanta 2002, 56, 365-373.

(38) Arbeloa, I. L. Dimeric and Trimeric States of the Fluorescein Dianion. Part 1.-Molecular Structures. J. Chem. Soc., Faraday Trans. 2 1981, 77, 1725-1733.

(39) Speiser, S.; Chisena, F. L. Optical Bistability in Fluorescein Dyes. Appl. Phys. B: Photophys. Laser Chem. 1988, 45, 137-144.

(40) Song, L.; Varma, C. A. G. O.; Verhoeven, J. W.; Tanke, H. J. Influence of the Triplet Excited State on the Photobleaching Kinetics of Fluorescein in Microscopy. Biophys. J. 1996, 70, 2959-2968.

(41) Song, L. L.; Hennink, E. J.; Young, I. T.; Tanke, H. J. Photobleaching Kinetics of Fluorescein in Quantitative Fluorescence Microscopy. Biophys. J. 1995, 68, 2588-2600.

(42) Velk, N.; Uhlig, K.; Vikulina, A.; Duschl, C.; Volodkin, D. Mobility of Lysozyme in Poly(L-lysine)/hyaluronic Acid Multilayer Films. Colloids Surf., B 2016, 147, 343-350.

(43) Xu, L.; Selin, V.; Zhuk, A.; Ankner, J. F.; Sukhishvili, S. A. Molecular Weight Dependence of Polymer Chain Mobility within Multilayer Films. ACS Macro Lett. 2013, 2, 865-868.

(44) Selin, V.; Ankner, J. F.; Sukhishvili, S. A. Nonlinear Layer-byLayer Films: Effects of Chain Diffusivity on Film Structure and Swelling. Macromolecules 2017, 50, 6192-6201.

(45) Aggarwal, N.; Altgärde, N.; Svedhem, S.; Zhang, K.; Fischer, S.; Groth, T. Study on Multilayer Structures Prepared from Heparin and Semi-synthetic Cellulose Sulfates as Polyanions and Their Influence on Cellular Response. Colloids Surf., B 2014, 116, 93-103.

(46) Aggarwal, N.; Groth, T. Multilayer Films by Blending Heparin with Semisynthetic Cellulose Sulfates: Physico-chemical Characterization and Cell Responses. J. Biomed. Mater. Res., Part A 2014, 102, 4224-4233.

(47) Muzzio, N. E.; Pasquale, M. A.; Gregurec, D.; Diamanti, E.; Kosutic, M.; Azzaroni, O.; Moya, S. E. Polyelectrolytes Multilayers to Modulate Cell Adhesion: A Study of the Influence of Film Composition and Polyelectrolyte Interdigitation on the Adhesion of the A549 Cell Line. Macromol. Biosci. 2016, 16, 482-495.

(48) Zhao, M.; Altankov, G.; Grabiec, U.; Bennett, M.; SalmeronSanchez, M.; Dehghani, F.; Groth, T. Molecular Composition of GAGcollagen I Multilayers Affects Remodeling of Terminal Layers and 
Osteogenic Differentiation of Adipose-derived Stem Cells. Acta Biomater. 2016, 41, 86-99.

(49) Muzzio, N. E.; Gregurec, D.; Diamanti, E.; Irigoyen, J.; Pasquale, M. A.; Azzaroni, O.; Moya, S. E. Thermal Annealing of Polyelectrolyte Multilayers: An Effective Approach for the Enhancement of Cell Adhesion. Adv. Mater. Interfaces 2017, 4, 1600126. 